Pain Physician. 2004;7:459-463, ISSN 1533-3159

\title{
USe of Radiopaque Contrast Agents for the Interventional Pain Physician
}

\author{
Pradeep Chopra MD, and Howard Smith MD
}

Intravenous contrast (IV) agents are used by Interventional Pain Management specialists for accurate localization of needle positions and spread of contrast agents to delineate pertinent anatomy. IV contrast agents are classified chemically as non-ionic or ionic monomers and dimers. Non-ionic IV contrast agents are more hydrophilic which makes them less neurotoxic. They also have a lower osmolality and produce fewer side effects. The desirable properties of IV contrast agents include satisfactory radiopacity, stability, pharmacological inertness and minimum sensitization.

In recent years, pain specialists have started using fluoroscopy units and radiologic adjuncts more and more often for accurate localization of needle position and the spread of contrast medium before injecting a local anesthetic, steroid or neurolytic agents. There is very little information in the pain management literature on the use of contrast agents. This article will address some of the pertinent issues relevant to the use of contrast agents for interventional pain procedures.

The amount of contrast medium which is utilized in interventional pain procedures is 'dwarfed' by comparison with the larger volumes used for interventional and diagnostic radiology. Even a perineural injection of contrast medium can be inadvertently injected intravenously or intrathecally. The clinical con-

From Boston University Medical Center, Boston, MA and Albany Medical College, Albany, NY

Address Correspondence: Pradeep Chopra, MD, 102 Smithfield Ave, Pawtucket, RI 02860

Disclaimer: No external funding was provided in preparing this manuscript.

Conflict of Interest: None

Acknowledgement:

Manuscript received on 04/30/04

Revision submitted on 08/04/04

Accepted for publication on 09/04/04
Physicians must obtain an informed consent for use of IV contrast agents prior to the procedure. A detailed history of allergies to iodine containing foods is a contraindication to using IV contrast agents.

Reactions to IV contrast agents may produce mild to severe symptoms. Facilities where IV contrast agents are administered must be well equipped with resuscitation equipment, medications and available trained staff. Pretesting with a small intradermal dose or subcutaneous injections, will not predict adverse reactions. Premedication with antihistamines, anticholinergics and di- azepam is less effective than with corticosteroids. Pretesting cannot be relied upon to predict severe reactions and may itself be hazardous for the patient.

The frequency of renal insult is especially high with pre existing azotemia, diabetes mellitus or elevated serum creatinine. Patients over the age of 65 years, with multiple myeloma, hypertension or hyperuricemia are at increased risk for developing nephrotoxicity.

Keywords: Intravenous contrast agents, iodine, interventional pain, ionic contrast agents, non-ionic contrast agents sequences of larger volumes of contrast agents used during diagnostic and interventional radiology procedures usually do not apply to the small amounts used for interventional pain clinic procedures, nevertheless it is important that the Pain Specialist keep in mind the potential effects of contrast medium on neural tissue and its systemic effects. The judicious use of contrast medium helps localize precise anatomic locations, may prevent aberrant spread of injectate, and may also reduce fluoroscopic time. When used with CAT scanning, contrast medium may be administered either orally or intravascularly, to enhance the image quality of the gastrointestinal tract and the vasculature thus facilitating precise needle localization.

\section{Classification of Contrast Agents}

Broadly, radiocontrast agents can be classified (1) as positive contrast agents and negative contrast agents. Positive contrast agents absorb x-rays and produce a darker shadow of the tissue to be visualized against the surrounding tissues, on the fluoroscopic screen and lighter shadows on the x-ray film. Negative contrast agents are more transparent to $\mathrm{x}$-rays than body tissue and produce a lighter shadow on the fluoroscopic screen. Gases such as air, nitrogen, carbon dioxide, helium, oxygen, nitrous oxide and xenon belong to this category. Pain Management practitioners most often use positive contrast agents or radiopaque agents; A small quantity of air maybe used to define tissue planes as in lumbar sympathetic blocks.

The properties (2) of radiopaque contrast agents that are desirable are:

1. Satisfactory radiopacity

2. Stable

3. Pharmacologically inert

4. Minimum sensitization

The chemical structure (3) of a contrast agent determines their properties and hence their clinical effects. The organic iodine compounds are extensively used and have a broad range of diverse applications. Contrast agents can be classified as:

a. Non ionic monomers and dimers

b. Ionic monomers and monoacidic dimers

\section{Non-Ionic Agents}

All agents contain an organic iodine compound that provides the contrast. Nonionic contrast agents are more hydrophilic than ionic agents. It is this greater hydrophilicity of the nonionic agents that 


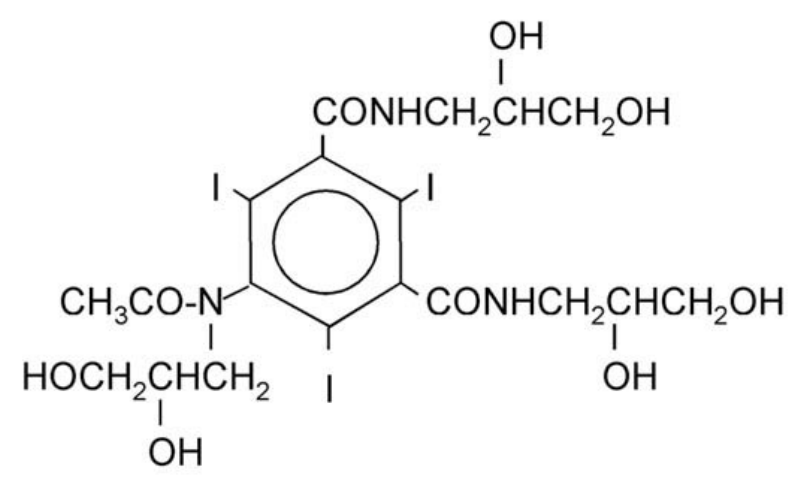

Fig 1. Chemical structure of Iohexol (brand name: Omnipaque), a nonionic contrast agent. The basic structure consists of a benzene ring with iodine atoms attached at the 2,4, and 6 positions. Addition of a COOH group makes it a salt. Non-ionic contrast agents have a fully substituted benzene ring. Agents that possess fewer carboxyl groups and evenly distributed hydroxyl groups are less neurotoxic

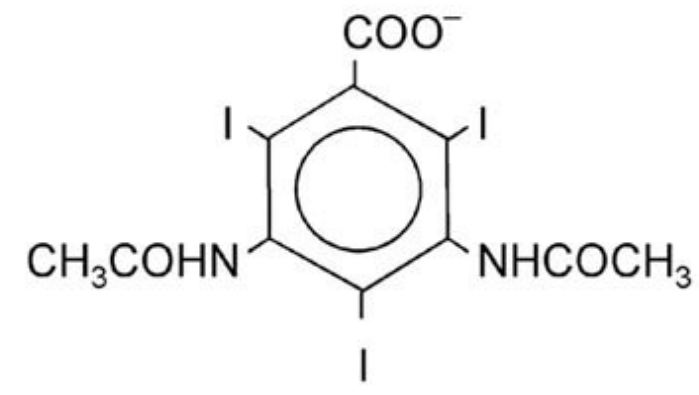

Fig 2. Chemical structure of Diatrizoate (brand names: e.g. Angiografin, Hypaque, Renografin, Urografin, Urovison), an ionic contrast agent.

reduces their subarachnoid and intravenous toxicity. They do not combine with either proteins or macromolecules, which makes them considerably less toxic than ionic contrast media. Nonionic contrast agents also have a low osmolality and produce fewer adverse effects. All myelography procedures as well as procedures with injection of contrast agents around neural tissue are best performed with nonionic agents.

Ionic Agents
The ionic compounds can be further subdivided into:

I. High osmolality

II. Low osmolality

Monomers have one iodinated benzene ring, while dimers have two iodinated benzene rings.

\section{GUIDELINES FOR THE USE OF Contrast Medium}

Interventional pain physicians must obtain an informed consent for use of contrast agents prior to the procedure.

\section{Table 1. Commonly used low osmolar contrast agents}

\begin{tabular}{lll|} 
Type & Generic name & Trade name \\
Non-ionic & lohexol & Omnipaque \\
& lopamidol & Isovue \\
& loversol & Optiray \\
& lodixanol & Visipaque \\
lonic & loxaglate & Hexabrix
\end{tabular}

The patient must be made aware of the benefits and risk of using a contrast agent. Fluoroscopy is indispensable for accurate needle localization and confirming spread of opaque contrast before injecting steroids, local anesthetics or neurolytic agents. They are also useful for delineating anatomy as in the spinal canal, facet joints, and sacroiliac joints. They are also useful for confirming position of epidural catheters and to rule out subdural or subarachnoid placement. Although, interventional pain physicians do not perform myelography, one must be aware of inadvertent intrathecal or intravascular administration of contrast dye.

Some practical considerations when using contrast agents -

- Make sure the syringe containing the contrast agent is labeled. In case of a doubt, you may place the syringe under the fluoroscope and identify it by the contrast (assuming there is 
only one contrast agent being used).

- A detailed history of allergies or previous administrations of contrast agents must be elicited prior to the procedure. A history of allergic reaction to iodine containing foods such as seafood is a contraindication to using contrast dyes.

- Contrast agents containing low concentrations of iodine appear safe with good imaging characteristics. These include contrast media containing iodine concentrations from $200 \mathrm{mg} / \mathrm{ml} \quad\left(\right.$ Isovue200 ${ }^{\circledR}$ ) to $300 \mathrm{mg} / \mathrm{ml}$ (Isovue ${ }^{\circledR} 250$, Isovue ${ }^{\circledR} 300$, Omnipaque ${ }^{\circledR} 300, \quad$ Optiray $^{\circledR} 300$, Oxilan ${ }^{\circledR} 300, \quad$ Ultravist ${ }^{\circledR} 300$ ). The recommended limit for iodine is 3 gm per day.

- Dilutions of up to $25 \%$ generally still produce good resolution for most procedures. The contrast agent may be diluted using normal saline.

- Store and use only non-ionic contrast agents in your facility. There is a substantial margin of safety with the use of non-ionic contrast agents.

- Ionic contrast agents have a higher incidence of neurotoxicity in the central nervous system (4). They can be used for peripheral nerves as in plexus blocks - however safety is optimized if there are no ionic contrast agents available in the pain procedure suite (if ionic contrast agents are not available then no ionic contrast agents could be mistakenly administered intrathecally).

- Metformin should be temporarily discontinued in patients undergoing procedures with IV contrast agents because it may result in acute alteration of renal function. Metformin is primarily excreted by the kidneys and any compromise of the renal function may result in higher levels of metformin with a risk of developing lactic acidosis.

\section{Adverse Reactions}

There is no accurate way to predict an adverse reaction. Some of the reactions can be attributed to allergic reactions (4) and some may be related to the chemical property of the agent, such as hyperosmolality or to chemotoxicity of the contrast agent. The toxicity can be classified as Chemotoxicity, Osmotoxicity and Ion toxicity.
Chemotoxicity refers to the molecular structure that binds to proteins and interacts with enzymes, cell membranes, cell compounds. Chemotoxic reactions may be related to osmotoxic side effects. They include cardiac effects such as arrhythmias, electrocardiogram changes and cardiac depression. Neurotoxicity to the spinal cord was first reported with the use of acetrizoate in high concentrations. Watersoluble angiographic contrast agents are more likely to cause damage to the spinal cord. There is a higher likelihood of damage to the spinal cord when the contrast agent is concentrated to the spinal cord as in inadvertent intrathecal administration, epidurogrpahy or transforaminal administration. Intracerebral or subarachnoid injection of either hypertonic or hypotonic non-ionic contrast agents in rats caused depression of the central nervous system. Procaine, Low Molecular Weight Dextran, $20 \%$ dextrose, and hypothermia may exert a protective effect against potential central nervous damage.

Osmotoxicity is related to the hyperosmolar nature of the contrast media. There is a significant difference in osmolality between the ionic and nonionic contrast media. Ionic contrast agents have a higher osmolality $(600-2400 \mathrm{mOsm} / \mathrm{kg}$ $\mathrm{H}_{2} \mathrm{O}$ ) compared to serum (300 mOsm/ $\mathrm{kg} \mathrm{H}_{2} \mathrm{O}$ )

Ion toxicity is the adverse reaction as a result of too high or too low ion concentration that interferes with cell function.

Adverse reactions to contrast agents can vary from mild to life threatening (6, $7)$. Most adverse effects are mild to moderate and do not require any treatment. The incidence of adverse effects is $5 \%$ to $12 \%$ in patients who receive ionic contrast agent. Some of the common reactions seen due to hyperosmolality are, pain on injection, sensation of warmth, vasodilatation (8). Hyperosmolality of the agent if it is high may also alter red blood cell plasticity and cause erythrocyte aggregation (9). High osmolality agents may promote sickling in patients who are homozygous for sickle cell disease. Additionally, high osmolality agents may cause damage to the vascular endothelium leading to release of endogenous vasoactive substances such as prostaglandins, kallikreins, and bradykinin (10).

The most frequently reported adverse reactions (11) to contrast media are headache, nausea, vomiting and musculoskeletal pain. These reactions usually oc-
Table 2. Symptoms of reactions to contrast agents

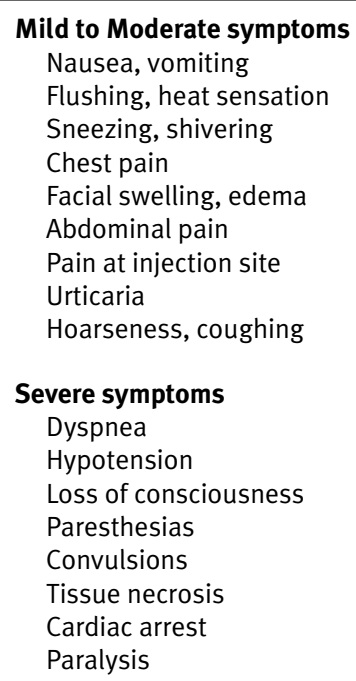

\section{Severe symptoms}

Dyspnea

Hypotension

Loss of consciousness

Paresthesias

Convulsions

Tissue necrosis

Cardiac arrest

Paralysis

cur 1 to 10 hours after injection and are mild to moderate in degree and will disappear in 24 hours. The headache after an intrathecal injection may persist for several days and is more common in patients not optimally hydrated. Backache, neck stiffness, numbness and paresthesias, leg or sciatic-type pain occurred less frequently, often in the form of a transient exacerbation of preexisting symptomatology. Other less frequent symptoms reported include facial neuralgia, tinnitus, sweating, and seizures. Peripheral neuropathies have been rare and transitory. They include sensory and/or motor or nerve root disturbances, myelitis, persistent leg muscle pain or weakness, or sixth nerve palsy or cauda equina syndrome. Muscle cramps, fasciculations or myoclonus, 'spinal convulsions', paralysis, or spasticity are unusual but can occur.

The use of contrast agents is associated with acute renal dysfunction (12, 13) especially with high osmolar ionized agents. The frequency of renal disturbance is especially high with pre existing azotemia, diabetes mellitus or elevated serum creatinine. Patients over the age of 65 years, with multiple myeloma, hypertension or hyperuricemia are at increased risk for developing nephrotoxicity.

Contrast agents, regardless of their ionic and osmolality differences, in the presence of $\mathrm{x}$-ray irradiation, may cause chromosome aberrations.

Most contrast agents are relatively hyperosmolar. When agents of high osmolality are administered it causes a 
net movement of water across cell membranes, which is known as osmotoxicity. To quantify osmotoxicity, a ratio between the imaging effects and osmotic effect of the contrast medium is used. The higher the ratio, the lower is the osmotoxicity. The number of iodine atoms present corresponds to the imaging effect. The osmotic effect relates to the number of particles present in an ideal solution. Low osmolar agents (ratio 3 to 6) are associated with lower neurotoxicity and preferred when used near nerve roots, major plexus or near the spinal cord. They are also associated with lower anaphylactoid reactions. Hence, nonionic, low osmolar contrast agents are preferred because of their hydrophilic nature; have low osmolality making them less osmotoxic.

\section{Precautions}

Pretesting patients with small intradermal dose or subcutaneous injections, will not predict adverse reactions. Premedication with antihistamines, anticholinergics and diazepam is less effective than with corticosteroids (14). Pretesting cannot be relied upon to predict severe reactions and may itself be hazardous for the patient.

Intrathecal administration of corticosteroids in combination with contrast agent is contraindicated. Use of medications that may lower the seizure threshold (phenothiazine derivates including those used for antihistamine properties; tricyclic antidepressants; MAO inhibitors; CNS stimulants; analeptics; antipsychotic agents) should be carefully evaluated. Caution should be used when administering contrast media to patients with increased intracranial pressure, history of convulsive disorder, severe cardiovascular disorder, chronic alcoholism, multiple sclerosis and elderly patients. Particular attention must be given to the state of hydration, concentration of medium, dose, and technique used in these patients.

Preparatory dehydration is dangerous and may contribute to acute renal failure in patients with advanced vascular disease, diabetic patients and in susceptible nondiabetic patients e.g. elderly with preexisting renal disease. Patients should be well hydrated prior to and following contrast media administration. Patients at increased risk include those with a history of a previous reaction to a contrast medium, patients with a known sensitivity to iodine per se, and patients with a known clinical hypersensitivity (bronchial asthma, hay fever, and food allergies). Additionally, especially if one is utilizing a large contrast load in a patient at high risk of nephrotoxicity from contrast (e.g. diabetic patients with pre-existing diabetic nephropathy). Strategies to protect renal function (15) should be considered including: bicarbonate infusion therapy, $\mathrm{N}$ acetyl cysteine (Mucomyst) therapy, and / or theophylline.

\section{Prophylactic Treatment to Avoid Reaction in Patients at Risk}

The general approach to managing a reaction can be summarized as the four Hs:

1. History - it must take into consideration any contraindications to contrast agents as in patients with allergic reactions to shellfish or diary products. Other risk factors are renal disease, significant cardiac disease, emotional state, paraproteinemias such as Multiple Myeloma. Diabetics on Metformin are at particular risk. Patients with hepatic dysfunction, renal dysfunction, alcohol abuse or severe congestive heart failure taking Metformin should not be administered contrast agents. There is a risk of precipitating lactic acidosis.

2. Hydration - it is important for patients to be well hydrated prior and after administration of the IV contrast agent. This is particularly important in patients prone to developing renal dysfunction.

3. Have expertise and equipment ready - be prepared to handle any serious adverse effect. This includes resuscitation equipment, drugs and trained personnel should be readily available.

4. Heads up - evaluate patients' general condition, be aware of any specific contraindications and respond promptly to the first sign of a reaction.

Prophylactic treatment in patients at risk for developing a reaction may be instituted. However, despite prophylactic therapy be aware that some reactions, although usually less catastrophic in nature - may occur. According to the Lasser (13, 14) approach, administer corticosteroid prior to using ionic contrast agents. Some of the recommendations are:

1. Corticosteroid and antihistamine combination - prednisone $50 \mathrm{mg}$ by mouth at 13 hours, 7 hours and 1 hour before contrast medium injection, with diphenhydramine (Benadryl ${ }^{\circledR}$ ) $50 \mathrm{mg}$ intravenously, intramuscularly or by mouth 1 hour prior to the contrast agent.

2. Corticosteroid alone Methylprednisolone $32 \mathrm{mg}$ by mouth at 12 hours and 2 hours prior to administering the contrast agent.

\section{Conclusion}

The choice of which contrast agent to use is left to the individual pain physician. It is important that he or she have a thorough understanding of the characteristics of various contrast agents. They are invaluable as a tool in interventional pain practice and have vastly improved outcomes and satisfaction from procedures. The newer agents have a higher safety profile. It is important that pain physicians understand and recognize any potential adverse reactions and be prepared to manage them.

\section{Author Affiliation \\ Pradeep Chopra, MD \\ Assistant Professor \\ Boston University Medical Center \\ Pain Management Center \\ 102 Smithfield Ave \\ Pawtucket, RI 02860 \\ E-mail: painri@yahoo.com \\ Howard Smith, MD \\ Academic Director of Pain Manage- ment \\ Dept. of Anesthesiology \\ Albany Medical College \\ 47 New Scotland Avenue \\ Albany, NY 12208 \\ E-mail: smithh@mail.amc.edu}

\section{REFERENCES}

1. Manual on Contrast Media, $4^{\text {th }}$ edition, 1998. American College of Radiology

2. Speck U. X-Ray contrast media. Schering, Berlin, 1987.

3. Peng CT. Radiopaques in Burger's Medicinal Chemistry and Drug Discovery, $5^{\text {th }}$ edition, Therapeutic agents.

4. Bohn HP, Reich L, Suljaga-Petchel K, (1992) Inadvertent intrathecal use of ionic contrast media for myelography AJNR 13: 1515-1519

5. Bettmann MA, Holtzer J, Trombley S. Contrast related risk management issues. $\mathrm{Ra}$ diology 1990; 175:629-631.

6. Bettmann MA, Heeren T, Greenfield A et al. Adverse events with radiographic contrast agents: results of SCVIR contrast agent registry. Radiology 1997; 203:611-620. 
7. Thomsen HS, Dorph S. High-osmolar and low-osmolar contrast media: an update on frequency of adverse drug reactions. Acta Radiol 1993; 34:205-209.

8. Wolf GL, Arenson RL, Cross AP. A prospective trial of ionic vs nonionic contrast agents in routine clinical practice: comparison of adverse effects. AJR 1989; 152:939-944.

9. Curry NS, Schabel SI, Reiheld CT et al. Fatal reactions to intravenous nonionic contrast material. Radiology 1991; 178:361362.

10. Almen T. The etiology of contrast medium reactions. Invest Radiol 1994; 29:37-45.

11

Wolf GL, Mishkin MM, Roux SG et al. Comparison of the rates of adverse drug reactions: ionic contrast agents, ionic agents combined with steroids, and nonionic agents. Invest Radiol 1991; 26:404-410.

12. Lalli AF. Mechanisms of contrast media reactions II. In Katzberg R (ed). The Contrast Media Manual. Williams \& Wilkins, Baltimore, 1992: 166-170.

13. Lasser EC. Mechanisms of contrast media reactions III. In Katzberg $\mathrm{R}$ (ed). The Contrast Media Manual. Williams \& Wilkins,
Baltimore, 1992: 171-179.

14. Lasser EC. Pretreatment with corticosteroids to prevent reactions to IV contrast material: overview and implications. $A J R$ 1988; 150:257-259.

15. Rosati G, de Haen C. Considerations on the research and development of new $X$ ray contrast media: A critical appraisal. In Katayama H, Brasch RC (eds). New Dimensions of Contrast Media Int. Congress Series 976. Excerpta Medica, Amsterdam, 1991, pp 29-34. 
